\title{
EL TURISMO GASTRONÓMICO COMO EXPERIENCIA CULTURAL. EL CASO PRÁCTICO DE LA CIUDAD DE CÁCERES (ESPAÑA) ${ }^{1}$
}

\author{
José Manuel Hernández-Mogollón \\ Universidad de Extremadura \\ jmherdez@unex.es \\ Elide Di-Clemente \\ Universidad de Extremadura \\ ediclemente@unex.es \\ Tomás López-Guzmán \\ Universidad de Córdoba. Campus de Excelencia Internacional Agroalimentario, ceiA3 \\ tomas.lopez@uco.es
}

\section{RESUMEN}

La valorización de las relaciones que unen la gastronomía y la cultura ha dado vida a un proceso de patrimonialización de los alimentos que se afirman como elementos tangibles de la cultura local y ofrecen nuevas posibilidades de diversificación para los destinos turísticos. El objetivo de este artículo es definir el perfil del turista gastronómico actual subrayando las conexiones entre la motivación gastronómica y la cultural. Se proponen los resultados de un estudio empírico desarrollado en la ciudad de Cáceres que demuestran correlaciones positivas entre la gastronomía y la cultura.

Palabras clave: turismo gastronómico, cultura, identidad local, motivación, Cáceres.

\section{ABSTRACT}

The importance recognized to the relationships that link gastronomy and culture has conferred to foodstuffs the status of tangible elements of local cultural heritage. As such, they

Fecha de recepción: junio 2013.

Fecha de aceptación: febrero 2014.

1 Este trabajo es uno de los resultados del Proyecto de Investigación de la Universidad de Extremadura (83 LOU): «La gastronomía de alta gama, de lujo o de calidad como eje potenciador del turismo cultural y de sensaciones en Extremadura». El investigador principal del proyecto es el Dr. José Manuel Hernández Mogollón y la entidad financiadora la Fundación Caja Extremadura. 
offer new possibilities for the diversification of tourist destinations. The aim of this paper is to define the profile of the current gastronomic tourist, highlighting the connections between the gastronomic and cultural motivation. The results of an empirical study conducted in the city of Cáceres show positive correlations between gastronomy and culture.

Keywords: culinary tourism, culture, local identity, motivation, Cáceres.

\section{INTRODUCCIÓN}

El turismo se caracteriza, cada vez más, como una actividad que proporciona al viajero una oportunidad de enriquecimiento personal y cultural (Ávila y Barrado, 2005). La movilidad turística pasa de ser un mero desplazamiento físico a identificarse como una experiencia introspectiva. Así, empiezan a desempeñar un papel importante todos aquellos elementos de la oferta turística de un destino que permiten al viajero experimentar este crecimiento cultural a lo largo de su viaje. Las tradiciones locales, los hábitos de los residentes, los estilos de vida y la gastronomía típica se identifican como los principales factores de diversificación de los destinos, capaces de impresionar al viajero e influenciar sus preferencias de compra. La gastronomía, entre otros, está afirmándose como un elemento importante de diversificación y como una herramienta de definición de marca y de imagen de un territorio, un país y una cultura (Hjalager, 2010). Las asociaciones entre los platos de la cocina local y su territorio de referencia son automáticas. En los últimos años, el turismo ha empezado a reconocer el importante poder de atracción y comunicación de los productos gastronómicos, hasta el punto de convertirlos en verdaderos atractivos turísticos (Folgado et al., 2011; Kivela y Crotts, 2006). Alrededor de las peculiaridades de la cocina local se desarrollan rutas, paquetes turísticos, propuestas e iniciativas temáticas que ponen a la gastronomía y a los productos de la tierra en el centro de las estrategias de desarrollo y de promoción de un determinado territorio o destino. La comida se está afirmando como un elemento de la cultura local y como la expresión de la historia, las costumbres y las tradiciones de una comunidad o una cierta área geográfica (Kivela y Crotts, 2006; Lee y Crompton, 1992).

La cultura, conjuntamente con la religión y otras caracterizaciones sociodemográficas, representa uno de los principales factores que determinan la aceptación de determinados productos dentro de la alimentación, así como su apreciación en término de gustos y sabores (Mak et al., 2012a). Así, la cultura determina y moldea la historia culinaria de un pueblo, la define y, a través de la misma, se expresa y se convierte en algo tangible, sensorial y placentero. Es esta última connotación que permite a los platos típicos y a los alimentos locales convertirse en verdaderos productos turísticos y factores motivacionales del viaje. Hoy la gastronomía mueve categorías de viajeros cada vez más amplias (Acevin, 2012; Fox, 2007; Segittur, 2010).

Fox (2007), con la expresión foodies, identifica a todos aquellos turistas que se demuestran sensibles a los patrimonios alimentarios de los destinos que visitan y que entienden la gastronomía como una experiencia compleja y cultural. Así, el producto típico o el plato tradicional de la cultura gastronómica local se eleva a testimonio de la identidad local y responde a una función de comunicación cultural y ya no sólo de satisfacción de una necesidad de alimentación. 
El principal objetivo de este artículo es definir el perfil del turista gastronómico actual, haciendo particular hincapié en las profundas relaciones que unen la motivación gastronómica y la cultural, para esta categoría de consumidores turísticos. Con esta finalidad, se ha llevado a cabo un estudio empírico en la ciudad de Cáceres mediante una labor de encuestación, a través de un cuestionario personal estructurado, dirigido a los turistas que se encontraban disfrutando de la gastronomía local en los restaurantes típicos de la ciudad. La estructura de este artículo se basa en cinco apartados. Tras esta introducción, en el segundo apartado se define el concepto de turismo gastronómico, identificando su marco teórico de referencia y subrayando la creciente patrimonialización de los recursos alimentarios locales como nuevos atractivos turísticos. En el tercer apartado se presenta el escenario de la investigación y la metodología empleada. En el cuarto apartado se muestran los resultados alcanzados con el trabajo de campo. Los datos recopilados han sido tratados con análisis estadísticos univariantes y bivariantes (tablas de contingencia y correlaciones) que han permitido alcanzar algunas conclusiones fundamentales del estudio que se reflejan en el quinto apartado. Finalmente, se presenta la bibliografía utilizada.

\section{DEFINICIÓN DE TURISMO GASTRONÓMICO}

La literatura identifica la gastronomía como un factor determinante en el éxito turístico de un destino, reconociendo los abundantes beneficios que proceden de una identidad culinaria consolidada y estructurada para los turistas (Belisle, 1983; Faulkner et al., 1999;Fox, 2007; Kivela y Crotts, 2006; Ramos et al., 2004;Reynolds, 1993). No obstante, son muy escasos los estudios encaminados a descubrir cuál es el peso que ejerce la gastronomía en la experiencia turística de los viajeros (Kivela y Crotts, 2006). El presente artículo encuentra en estas consideraciones su principal justificación.

En cuanto a su definición científica, Kivela y Crotts (2006) afirman que la gastronomía es una actividad compleja e interdisciplinar que ahonda sus raíces en las culturas de las mayores civilizaciones clásicas.

Al ser una tipología turística en crecimiento, el turismo gastronómico está definido por una literatura todavía incipiente (Hjalager y Richards, 2002; Long, 2003).

Kivela y Crotts (2006) hacen hincapié en el error que se comete al considerar la gastronomía exclusivamente como el arte del cocinar y del buen comer. Esta acepción resulta limitativa, sobre todo si admitimos la carga cultural que se esconde detrás de los alimentos y, aún más, si consideramos sus relaciones con el turismo. Según Schlüter (2009) el turismo gastronómico corresponde a aquella actividad turística de la cual participan personas cuya elección del destino a visitar se encuentra muy influenciada por una motivación culinaria. Millán et al. (2010), por otro lado, consideran el turismo gastronómico como el realizado por aquellas personas que visitan un lugar atraídas por su gastronomía o por sus productos agro-alimentarios. Leal (2011: 16) considera que el turismo gastronómico«...resulta en la actualidad como una de las mejores expresiones de la sociedad postindustrial en donde el hecho de ver, oler y degustar, se convierte en una actividad completamente experiencial que es generada por esas industrias (productores, transformadores, restauradores) cuya principal finalidad o producto es poder otorgarle al turista una experiencia que pueda ser vivida a través de la comida o la bebida». 
En general, podemos afirmar que la gastronomía típica de un destino es el resultado de una cultura histórica y social del mismo (Kivela y Crotts, 2006). Esta puede ser latente y estar mortificada ante la determinada afirmación de costumbres alimentarias internacionales y globalizadas (Fox, 2007; Ritzer, 1995), o bien mantenerse original y diferente con respecto a otras gastronomías. En este último caso, el turismo gastronómico representa una tipología turística que, a través de la valorización de los recursos culinarios locales, expresa autenticidad, espectacularidad, sensorialidad, erudición del consumidor, experiencias y coproducción del servicio. Roden (2003) afirma que las costumbres alimentarias representan una parte fundamental y tangible de la cultura de un pueblo y un legado para las futuras generaciones. Molz (2007), por otra parte, afirma que el turismo gastronómico es una práctica exploratoria que lleva a conocer otros lugares y hábitos y que se concretiza en la degustación de platos y productos diversos a los de la cotidianidad. En este sentido la gastronomía es parte de la identidad local y en ella se refleja la cultura de un pueblo y la vida de las personas.

\section{II.1. Marco teórico del turismo gastronómico}

Desde una perspectiva teórica, la literatura científica aborda el tema del turismo gastronómico desde dos ámbitos y tipologías turísticas consolidadas como son: el turismo rural y el turismo cultural (Millán y Agudo, 2010). En cuanto a la primera, varios autores investigan el fenómeno del turismo gastronómico y de la reciente atención otorgada a la tipicidad alimentaria por los turistas, a partir de su complementariedad con las actividades rurales. Sidali et al. (2013) afirman que las zonas rurales representan los escenarios ideales para el desarrollo de actividades e iniciativas gastronómicas, debido a la fuerte identidad culinaria que tradicionalmente caracteriza estos territorios y, al mismo tiempo, al hecho que en estas zonas se encuentran los centros de producción y elaboración de los alimentos autóctonos. Dentro de esta categorización, quedarían incluidas también las consideraciones acerca de las relaciones que unen la gastronomía y la tipología turística de «sol y playa». Los autores que han profundizados en estas últimas (Andreu y Verdú, 2012; Díaz, 2008) identifican el turismo gastronómico, concebido en la mayoría de los casos en sus sub-productos del oleoturismo y el enoturismo, como una oportunidad para diversificar los destinos maduros de sol y playa y otorgando cierta relevancia turística a las regiones rurales del interior marítimo. A través de la valorización de los patrimonios gastronómicos se pretende, pues, descongestionar los destinos turísticos de litoral y diversificar la oferta local, direccionando los flujos y los ingresos turísticos hacía las campiñas rurales cercanas.

Por otra parte, otros autores (Dos Santos y Antonini, 2004; Kivela y Crotts, 2006; LópezGuzmán y Sánchez-Cañizares, 2008; Mak et al., 2012a; Moltz, 2007; Richards, 2002; Ritzer, 1995; Roden, 2003; Sánchez-Cañizares y López-Guzmán, 2011) conceptualizan la gastronomía dentro del marco más amplio y consolidado del turismo cultural, subrayando las fuertes implicaciones culturales que subyacen entre la gastronomía y el turismo. A colación Kivela y Crotts (2006) defienden que aquellos consumidores interesados en los alimentos, normalmente se interesan de aspectos multidisciplinares, relacionados con la cocina típica y la gastronomía, subrayando la curiosidad cultural del turista gastronómico.

Así, el turismo culinario o gastronómico debe inevitablemente ser considerado como la expresión de una nueva tendencia cultural del consumidor. Este último se interesa por los alimentos y los platos típicos con la finalidad de conocer el entramado cultural que los ha 
determinado y la historia que existe a su alrededor. En este artículo, reconocemos el turismo gastronómico como una práctica cultural y la gastronomía típica como un exclusivo patrimonio sensorial y experiencial.

\section{II.2. Turismo gastronómico y cultura: patrimonialización de los productos típicos locales}

Espeitx (2004) apunta que al patrimonio alimentario se le reconoce un papel importante en tipos de turismo como el agroturismo, el turismo rural, el ecoturismo, el turismo cultural, etc. y también forma parte de museos y exposiciones, centros de interpretación, rutas, ferias o campañas gastronómicas.

En algunos casos, el producto gastronómico se convierte en el principal o único atractivo de un destino alrededor del cual se desarrollan campañas de comercialización y de promoción del mismo con su consecuente traducción en una rentable propuesta turística. En este sentido, Kivela y Crotts (2006) nos ofrecen el ejemplo de territorios como La Toscana italiana, con sus famosas cooking experiences y sus tours culinarios, o Lyon en Francia, cuya gastronomía se ha hecho famosa gracias a chefs de renombre internacional.

Según Kivela y Crotts (2006), la gastronomía se está convirtiendo en un factor importante en el desarrollo de un turismo de nicho y de destinos especializados en este tipo de recurso. Los estudios demuestran que la cuota de gasto que los turistas dedican a la comida equivale a un tercio de su gasto total, lo cual le identifica como segmento turístico extremadamente atractivo para los destinos (Fox, 2007; Hall y Sharples, 2003;Telfer y Wall, 2000). Algunos autores mantienen que la cultura culinaria representa una importante fuente de identidad en la sociedad postmoderna (Richards, 2002), hasta el punto de convertirse en una manera de defenderse de la creciente homologación gastronómica que se está produciendo tras el fenómeno de la globalización y de la denominada MacDonaldización (Ritzer, 1995) de los platos y de las comidas (Feo, 2005;Mak et al., 2012b). Mak et al. (2012b) identifican, como efecto positivo de la homologación de las culturas provocada por la globalización, la creciente atención que se está reservando a las tradiciones locales, los hábitos y las costumbres tradicionales y, entre ellos, la cultura gastronómica de un territorio, aglutinando en este concepto los procesos de producción tradicionales, los alimentos locales, las recetas y las formas típicas de elaboración de los alimentos.

La literatura respalda las fuertes conexiones que subyacen entre alimentación y turismo. Actualmente existen varias líneas de investigación abiertas para profundizar en las mismas (Campón et al., 2011). No obstante, cabe destacar que es una área de estudio nueva y joven (Kivela y Crotts, 2006).

En el viaje, la gastronomía se identifica como una parte integrante de la experiencia turística ya que implica la posibilidad de saborear alimentos y platos distintos a los de nuestra cotidianidad o, simplemente, enseña una nueva forma de disfrutar de los mismos (Quan y Wang, 2004). De esta manera, la curiosidad gastronómica se considera como factor motivacional añadido que lleva a los turistas a querer acercarse a nuevos estilos culinarios y de vida (Dos Santos y Antonini, 2004). Quan y Wang (2004) identifican en este aspecto la componente experiencial de la comida y de la gastronomía como producto turístico y como principal atractivo de un destino. De esta forma, los productos agroalimentarios, así como los vinos de la tierra, se convierten en un activo patrimonial extremadamente importante. 


\section{ESCENARIO Y METODOLOGÍA}

Cada vez más, los destinos utilizan la gastronomía como una fuente de nuevos productos y actividades para atraer turistas. La investigación en el sector del turismo gastronómico surge como respuesta a los constantes cambios del mercado y de las preferencias de los consumidores. El análisis de cómo los turistas ven y evalúan sus experiencias de viajes gastronómicos constituye una base esencial para el desarrollo y la promoción de las rutas turísticas (Stewart et al., 2008).

En este artículo se presentan los resultados de una investigación empírica desarrollada en la ciudad de Cáceres, situada en la región de Extremadura, España.

Extremadura se identifica como un territorio que puede presumir de un patrimonio abundante y singular. Los últimos datos divulgados por la Consejería de Fomento, Vivienda, Ordenación del Territorio y Turismo de la Junta de Extremadura demuestran el fuerte impacto que mantiene el turismo sobre la economía local y la necesidad de fomentar el crecimiento y la cualificación de este sector que ya representa el 4,3\% del PIB regional y se cuantifica en más de 700 millones de euros (Ortega et al., 2012). Sus recursos histórico-artísticos y naturales convierten la región en un escenario turístico único. En relación con los flujos de turistas que visitan la ciudad de Cáceres, cabe destacar que se trata principalmente de viajeros nacionales, quedando los turistas extranjeros, en una minoría.

En las Tablas 1 y 2 se muestra la evolución del número de viajeros y de pernoctaciones registradas en el periodo 2007-2011 en la ciudad de Cáceres.

Tabla 1

EVOLUCIÓN DEL NÚMERO DE VIAJEROS EN LA CIUDAD DE CÁCERES. PERIODO 2007-2011

\begin{tabular}{|c|c|c|c|}
\hline AÑO & TOTAL & ESPAÑOLES & EXTRANJEROS \\
\hline 2007 & 213.383 & 187.980 & 25.403 \\
\hline 2008 & 201.187 & 176.357 & 24.830 \\
\hline 2009 & 206.973 & 183.021 & 23.952 \\
\hline 2010 & 215.490 & 192.783 & 22.706 \\
\hline 2011 & 233.053 & 202.811 & 30.242 \\
\hline
\end{tabular}

Fuente: Instituto Nacional de Estadística -INE- (2012).

Tabla 2

EVOLUCIÓN DEL NÚMERO DE PERNOCTACIONES EN LA CIUDAD DE CÁCERES. PERIODO 2007-2011

\begin{tabular}{|c|c|c|c|}
\hline AÑO & TOTAL & ESPAÑOLES & EXTRANJEROS \\
\hline 2007 & 342.243 & 300.590 & 41.653 \\
\hline 2008 & 336.971 & 295.418 & 41.553 \\
\hline 2009 & 326.969 & 286.717 & 40.252 \\
\hline 2010 & 341.579 & 306.930 & 34.644 \\
\hline 2011 & 368.701 & 326.394 & 42.306 \\
\hline
\end{tabular}

Fuente: Instituto Nacional de Estadística -INE- (2012). 
El secular aislamiento de la ciudad de los sistemas de producción industrializados y una economía tradicionalmente asentada en las actividades del sector primario, le han permitido conservar sus tradiciones y sus identidades territoriales. Estos aspectos la convierten en un territorio auténtico y peculiar, donde se hace necesario un planteamiento de desarrollo turístico original y, por ciertos aspectos, discreto, capaz de valorizar las autenticidades locales, poniéndolas al centro del sistema de oferta turística (Calzati, 2009; Lancerini, 2005; Lanzani, 2005). Entre los principales elementos de identidad de un territorio debemos incluir, sin duda, la gastronomía que es un importante vehículo de la cultura local (Roden, 2003). Extremadura además de contar con una cocina rica y variada, caracterizada por platos típicos y recetas tradicionales, cuenta con materias primas de gran calidad. Tanto los productos de la tierra como los cárnicos y los lácteos extremeños se distinguen por ser elaborados según procesos de producción y transformación tradicionales. Sus peculiaridades gastronómicas nos ofrecen la oportunidad de aprovechar las sinergias existentes entre el sector primario y el turismo abriendo el paso a una propuesta turística auténtica y fiel al territorio (Ortega et al., 2012).

En cuanto a la oferta gastronómica, el territorio objeto de estudio destaca por el uso de productos autóctonos, muchos de los cuáles están amparados bajo certificaciones de calidad (Denominaciones de Origen -DD.OO.- e indicaciones Geográficas Protegidas -II.GG. PP.-). Entre las DD. OO. destacamos las siguientes: Jamón Dehesa de Extremadura, Cereza del Jerte, Aceite Gata-Hurdes, Miel de Villuercas-Ibores, Torta del Casar, Queso Ibores y Pimentón de la Vera. Por otro, el sello de I.G.P. lo ostentan la Ternera y el Cordero de Extremadura (Ortega et al., 2012). En la mayoría de los casos, se trata de productos elaborados y producidos en las zonas rurales próximas a la ciudad de Cáceres y que adquieren sus especiales propiedades organolépticas gracias al clima, los procesos productivos tradicionales y el emplazamiento de esta determinada zona geográfica. En este sentido, la carne de caza representa uno de los elementos distintivos de la tradición alimentaria y gastronómica de la ciudad, siendo la base de muchas recetas y platos típicos (codornices, perdiz, venado, jabalí, conejos y liebres) (Ortega et al., 2012). No obstante, el producto estrella de la tradición culinaria local se reconoce en el cerdo ibérico y en todos sus derivados (jamón, embutidos y productos cárnicos).

Además de productos autóctonos de calidad, Cáceres ofrece una amplia variedad de establecimientos de restauración que destacan por sus manjares, su emplazamiento y el talento de sus cocineros. Algunos de ellos han sido galardonados por las principales guías gastronómicas nacionales e internacionales, como las Guías Repsol y Michelin (un establecimiento con dos estrellas Michelin y tres soles Repsol y diez restaurantes recomendados en las dos guías) (Guía Michelin, 2012; Guía Repsol, 2012). La mayoría de dichos restaurantes se encuentran ubicados en la parte histórica de la ciudad y son elegidos normalmente por aquellos consumidores que buscan conciliar la experiencia gastronómica con el disfrute de un escenario monumental singular, como es el casco antiguo de Cáceres, declarado Patrimonio de la Humanidad por la UNESCO en 1986.

A partir de estas consideraciones, se advierte la necesidad de investigar la demanda de turismo gastronómico. La medición, en términos cuantitativos, de los viajeros interesados en los recursos culinarios locales y el conocimiento de su actual percepción de la gastronomía, nos permite otorgar la justa importancia a los patrimonios gastronómicos locales 
y detectar las acciones y los acondicionamientos a la oferta turística, necesarios para conseguir captar y satisfacer las necesidades de este atractivo segmento de consumidores.

A nivel metodológico, cabe especificar que se ha desarrollado un trabajo empírico con encuestas personales estructuradas, dirigidas a los turistas que se encontraban disfrutando de la gastronomía local en los restaurantes de comida típica o tradicional de la ciudad de Cáceres. El cuestionario en papel ha sido la herramienta utilizada para la recogida de datos primarios. Con anterioridad al trabajo de campo, se ha sometido el cuestionario a 12 expertos entre académicos y profesionales del sector cuyas sugerencias y correcciones han contribuido a elaborar la versión definitiva del cuestionario. La población objetivo se ha identificado en los turistas o excursionistas, españoles o extranjeros, que se encontraban en los restaurantes turísticos de la ciudad de Cáceres en los meses de abril y mayo de 2012. Para lo que se refiere a la técnica de muestreo se ha optado por una técnica no probabilística y de conveniencia. Los encuestados han sido seleccionados por intercepción, intentando abarcar distintas capas sociodemográficas hasta conseguir una muestra total de 720 individuos. En la Tabla 3, propuesta a continuación, se detallan los aspectos técnicos de la investigación.

Tabla 3

FICHA TÉCNICA DE LOS CUESTIONARIOS

\begin{tabular}{|l|l|}
\hline Universo & Turistas y excursionistas de la ciudad de Cáceres \\
\hline Ámbito & Municipal, Cáceres, (España) \\
\hline Método de recogida de información & Encuesta personal con cuestionario estructurado \\
\hline Base de datos & No existe \\
\hline Unidad muestral & Turistas y excursionistas de la ciudad de Cáceres \\
\hline Tamaño de la población & No definido \\
\hline Tamaño de la muestra & 720 \\
\hline Muestreo & No probabilístico de conveniencia \\
\hline Nivel de Confianza & $95 \%$ \\
\hline Error Muestral & $+/-3,7 \%$ \\
\hline Trabajo de campo & Abril y mayo de 2012 \\
\hline Número de entrevistas realizadas & 720 \\
\hline Respuestas válidas & Todas \\
\hline
\end{tabular}

Fuente: Elaboración propia.

Los datos recopilados se han tabulado y analizado con el programa estadístico SPSS Versión 19 y han sido tratados con análisis univariantes y bivariante (tablas de contingencias y correlaciones) para obtener los resultados.

\section{RESULTADOS DE LA INVESTIGACIÓN Y DISCUSIÓN}

El estudio cuantitativo acerca de la demanda de turismo gastronómico nos ha proporcionado una importante información en relación con el perfil del turista que visita un destino, siendo la gastronomía y la cultura variables motivacionales importantes. 
Antes de exponer los principales resultados del estudio, cabe destacar algunos aspectos de la muestra. La distribución por sexo es equilibrada, pues el 49,4\% de los encuestados está representado por hombres y el 50,6\% por mujeres. La mayoría de los individuos se encuentra entre los 40 y los 49 años $(23,8 \%)$, aunque no difieren mucho los porcentajes correspondientes a las franjas 30-39 (22,5\%) y 50-59 (23,4\%). Los niveles de formación son elevados ya que el $57,5 \%$ de la muestra declara tener estudios universitarios. Los encuestados proceden de 153 ciudades diferentes y 16 países. De manera coherente con los datos oficiales del INE (2012) acerca de los flujos de viajeros que visitan la ciudad de Cáceres (Véase Tablas 1 y 2), el estudio cuenta con un público de turistas esencialmente nacional, siendo el 90,8\% representado por españoles, y tan sólo el 9,2\% por extranjeros, destacando Francia y Portugal, como principales mercados emisores europeos, y Argentina como extraeuropeo. Es interesante notar que la mayoría $(30,9 \%)$ de los encuestados ha declarado tener unos ingresos mensuales superiores a los 2.000 euros, lo cual indica un elevado poder adquisitivo. Este último dato nos permite contrastar los datos de la literatura, según los cuales las destinaciones que ofrecen una gastronomía atractiva se disponen a recibir un segmento de consumidores pudientes y con un poder adquisitivo medio-alto (Fox, 2007).

En la Tabla 4, se presentan los principales datos sociodemográficos de la muestra.

Tabla 4

CARACTERÍSTICAS SOCIODEMOGRÁFICAS DEL TURISTA DE LA CIUDAD DE CÁCERES

\begin{tabular}{|l|l|l|l|}
\hline \multicolumn{1}{|c|}{ Variable } & \multicolumn{1}{c|}{ Ítem } & Frecuencia & \multicolumn{1}{c|}{$\%$} \\
\hline Sexo & Varón & 342 & 49,4 \\
& Mujer & 351 & 50,6 \\
\hline Edad & Menos de 30 años & 111 & 15,6 \\
$N=710$ & 30-39 años & 160 & 22,5 \\
& $40-49$ años & 169 & 23,8 \\
& $50-59$ años & 166 & 23,4 \\
\hline Procedencia & 60 años o más & 104 & 14,6 \\
N=699 & España & 635 & 90,8 \\
Formación & Resto & 64 & 9,2 \\
$N=701$ & Bachillerato o inferior & 209 & 29,8 \\
& F.P. & 89 & 12,7 \\
Ingresos & Universitaria & 403 & 57,5 \\
N=595 & Menos 1.000 $€$ & 108 & 18,2 \\
& $1.000-1.500 €$ & 158 & 26,6 \\
& $1.501-2.000 €$ & 145 & 24,4 \\
& Más de 2.000€ & 184 & 30,9 \\
\hline
\end{tabular}

Fuente: Elaboración propia.

En la Tabla 5 podemos observar aspectos relacionados con el comportamiento del turista en relación con la organización del viaje (con quién viaja, la estancia media y primera visita). 
Tabla 5

TIEMPO DE PERMANENCIA

\begin{tabular}{|l|l|l|l|}
\hline Variable & Ítem & Frecuencia & \% \\
\hline \multirow{5}{*}{ Estancia media } & No pernocta & 193 & 27,3 \\
\cline { 2 - 4 } & Pernocta 1 noche & 262 & 37,1 \\
\cline { 2 - 4 } & $3-7$ días & 197 & 27,9 \\
\cline { 2 - 4 } & Más de una semana & 55 & 7,8 \\
\hline \multirow{5}{*}{ Como viaja } & Solo & 38 & 5,4 \\
\cline { 2 - 4 } & Con compañeros/amigos & 241 & 34,0 \\
\cline { 2 - 4 } & Con pareja & 313 & 44,2 \\
\cline { 2 - 4 } & Con pareja e hijos & 76 & 10,7 \\
\cline { 2 - 4 } & Con hijos & 7 & 1,0 \\
\cline { 2 - 4 } & Otro & 33 & 4,7 \\
\hline \multirow{2}{*}{ Primera visita } & Si & 331 & 48,1 \\
\cline { 2 - 4 } & No & 357 & 51,9 \\
\hline
\end{tabular}

Fuente: Elaboración propia.

La mayoría de los encuestados $(37,1 \%)$ ha declarado pernoctar, por lo menos, una noche en el destino, denotando la tendencia a un turismo de fin de semana. Se trata principalmente de parejas $(44,2 \%)$ o de grupos de compañeros y amigos $(34,0 \%)$. En cuanto a la primera visita, no existen diferencias significativas entre los grupos de encuestados que han declarado estar repitiendo la visita a la ciudad de Cáceres y aquellos que la visitan por primera vez. Este dato pone de manifiesto que aproximadamente un $50 \%$ de los turistas que visitan Cáceres suelen repetir la visita en futuras ocasiones.

\section{IV.1. La gastronomía como elemento cultural motivador de la visita}

Los resultados relativos a la pregunta de motivación propuesta a los encuestados han puesto de manifiesto la importancia y el peso que ejercen los patrimonios cultural y gastronómico como principales atractivos de la ciudad.

En la Figura 1 podemos apreciar la valoración media para cada una de las motivaciones propuestas. Los datos nos permiten apreciar la relevancia que tienen la gastronomía típica y la cultura como atractivo turístico de la ciudad. La variable motivacional se ha medido con una escala Likert de 5 puntos, donde 1 corresponde a Nada Importante y 5 a Muy Importante.

La motivación cultural resulta la más destacada. El 47,2\% de los encuestados considera la posibilidad de aprender sobre la cultura y el patrimonio de Cáceres como un elemento motivacional muy importante, asignándole 5 puntos. Como se aprecia en la Figura 1, su valoración media es muy próxima al máximo $(4,07)$. El importante patrimonio monumental de la ciudad de Cáceres y su casco antiguo, declarado Patrimonio de la Humanidad por la UNESCO, no deja dudas sobre la vocación turística de este destino. No obstante, la gastronomía local se coloca en segunda posición con una valoración media de 3,9. Cabe destacar que más del 60\% de los turistas encuestados han señalado la gastronomía como motivación bastante importante (32\%) o muy importante $(37,3 \%)$. Es necesario detenernos sobre estos datos y profundizar en 
las posibles relaciones que unen la gastronomía y la cultura en un determinado destino turístico. Los datos corroboran la teoría expuesta anteriormente según la cual la gastronomía se cualifica, cada vez más, como parte de la cultura de un territorio (Dos Santos y Antonini, 2004; Fox, 2007; Quan y Wang, 2004; Roden, 2003; Sánchez-Cañizares y López-Guzmán, 2011).

Figura 1

IMPORTANCIA DE ELEMENTOS MOTIVACIONALES PARA LA VISITA DE LA CIUDAD DE CÁCERES

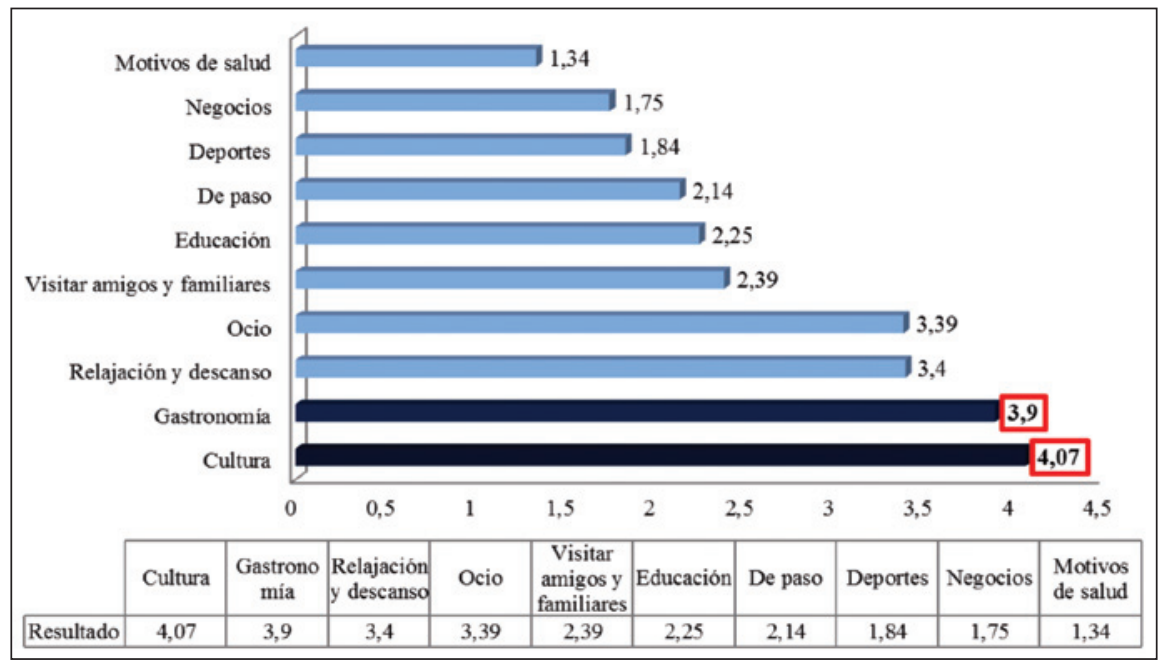

Fuente: Elaboración propia.

Los datos empíricos confirman una tendencia que asocia la motivación cultural con la gastronómica. No es una casualidad que las valoraciones más elevadas correspondan precisamente a estas dos variables. Observando la Tabla 6 podemos descubrir que para la mayoría de los turistas que consideran la cultura una motivación muy importante, también lo es la gastronomía. En general, se puede detectar una tendencia coherente en cuanto a la valoración que se ha asignado a los dos aspectos motivacionales considerados.

La asociación de estas dos motivaciones nos ofrece una importante información acerca de la percepción que mantienen los turistas que visitan Cáceres del patrimonio gastronómico local, y nos permite contrastar y confirmar el planteamiento teórico del turismo gastronómico. Varios autores (Fox, 2007; Quan y Wang, 2004; Roden, 2003) han afirmado la reciente tendencia de considerar la gastronomía como un elemento fundamental de la cultura de un destino y no simplemente como la respuesta a la necesidad fisiológica de alimentación. El hecho que el $51,1 \%$ de los encuestados que consideran muy importante la cultura haya reconocido la misma importancia a la gastronomía típica sugiere que, entre la demanda turística actual, existe un fuerte interés acerca de la cultura culinaria local, más allá de la finalidad alimentaria. En las buenas mesas y en el contacto con los hábitos culinarios y los alimentos más tradicionales, el turista busca un patrimonio a descubrir para su enriquecimiento personal, al igual que pasa con la visita de los elementos culturales más tradicionales como los monumentos, los museos, etc. 
Tabla 6

ANÁLISIS COMPARATIVO ENTRE MOTIVACIÓN GASTRONÓMICA Y CULTURAL

\begin{tabular}{|c|c|c|c|c|c|c|c|c|}
\hline & \multicolumn{5}{|c|}{ Disfrutar de la gastronomía típica } & \multirow[b]{2}{*}{ Total } \\
\hline & & & $\begin{array}{c}\text { Nada } \\
\text { importante }\end{array}$ & $\begin{array}{c}\text { Poco } \\
\text { importante }\end{array}$ & Importante & $\begin{array}{l}\text { Bastante } \\
\text { importante }\end{array}$ & $\begin{array}{c}\text { Muy } \\
\text { importante }\end{array}$ & \\
\hline \multirow{5}{*}{ 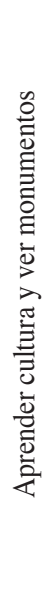 } & $\begin{array}{l}\text { Nada } \\
\text { importante }\end{array}$ & $\begin{array}{l}\text { Recuento } \\
\% \text { dentro } \\
\text { de cultura }\end{array}$ & $\begin{array}{r}11 \\
37,9 \%\end{array}$ & $\begin{array}{r}2 \\
6,9 \%\end{array}$ & $\begin{array}{r}6 \\
20,7 \%\end{array}$ & $\begin{array}{r}2 \\
6,9 \%\end{array}$ & $\begin{array}{r}8 \\
27,6 \%\end{array}$ & $\begin{array}{r}29 \\
100,0 \%\end{array}$ \\
\hline & $\begin{array}{l}\text { Poco } \\
\text { importante }\end{array}$ & $\begin{array}{l}\text { Recuento } \\
\% \text { dentro } \\
\text { de cultura }\end{array}$ & $\begin{array}{r}1 \\
4,8 \%\end{array}$ & $\begin{array}{r}1 \\
4,8 \%\end{array}$ & $\begin{array}{r}8 \\
38,1 \%\end{array}$ & $\begin{array}{r}4 \\
19,0 \%\end{array}$ & $\begin{array}{r}7 \\
33,3 \%\end{array}$ & $\begin{array}{r}21 \\
100,0 \%\end{array}$ \\
\hline & Importante & $\begin{array}{l}\text { Recuento } \\
\% \text { dentro } \\
\text { de cultura }\end{array}$ & $\begin{array}{r}5 \\
5,2 \%\end{array}$ & $\begin{array}{r}6 \\
6,3 \%\end{array}$ & $\begin{array}{r}36 \\
37,5 \%\end{array}$ & $\begin{array}{r}26 \\
27,1 \%\end{array}$ & $\begin{array}{r}23 \\
24,0 \%\end{array}$ & $\begin{array}{r}96 \\
100,0 \%\end{array}$ \\
\hline & $\begin{array}{l}\text { Bastante } \\
\text { importante }\end{array}$ & $\begin{array}{l}\text { Recuento } \\
\% \text { dentro } \\
\text { de cultura }\end{array}$ & $\begin{array}{r}6 \\
3,9 \%\end{array}$ & $\begin{array}{r}3 \\
1,9 \%\end{array}$ & $\begin{array}{r}32 \\
20,8 \%\end{array}$ & $\begin{array}{r}75 \\
48,7 \%\end{array}$ & $\begin{array}{r}38 \\
24,7 \%\end{array}$ & $\begin{array}{r}154 \\
100,0 \%\end{array}$ \\
\hline & $\begin{array}{l}\text { Muy } \\
\text { importante }\end{array}$ & $\begin{array}{l}\text { Recuento } \\
\% \text { dentro } \\
\text { de cultura }\end{array}$ & $\begin{array}{r}12 \\
4,4 \%\end{array}$ & $\begin{array}{r}10 \\
3,7 \%\end{array}$ & $\begin{array}{r}34 \\
12,5 \%\end{array}$ & $\begin{array}{r}77 \\
28,3 \%\end{array}$ & $\begin{array}{r}139 \\
51,1 \%\end{array}$ & $\begin{array}{r}272 \\
100,0 \%\end{array}$ \\
\hline \multicolumn{2}{|c|}{ Total } & $\begin{array}{l}\text { Recuento } \\
\% \text { dentro } \\
\text { de cultura }\end{array}$ & $\begin{array}{r}35 \\
6,1 \%\end{array}$ & $\begin{array}{r}22 \\
3,8 \%\end{array}$ & $\begin{array}{r}116 \\
20,3 \%\end{array}$ & $\begin{array}{r}184 \\
32,2 \%\end{array}$ & $\begin{array}{r}215 \\
37,6 \%\end{array}$ & $\begin{array}{r}572 \\
100,0 \%\end{array}$ \\
\hline
\end{tabular}

Fuente: Elaboración propia.

Tanto la intensidad como la significatividad estadística de la asociación que existe entre motivación gastronómica y cultural se ven corroboradas por el coeficiente de correlación de Pearson $(0,292)$ que demuestra la existencia de una correlación positiva y estadísticamente significativa $(\mathrm{p}=0.000)$ (Véase Tabla7).

Tabla 7

CORRELACIONES ENTRE LAS MOTIVACIONES CULTURA Y GASTRONOMÍA

\begin{tabular}{|l|l|r|r|}
\hline \multicolumn{2}{|c|}{} & \multicolumn{1}{|c|}{ Cultura } & \multicolumn{1}{c|}{ Gastronomía } \\
\hline \multirow{4}{*}{ Cultura } & Correlación de Pearson & 1 & $0,292^{* *}$ \\
& Sig. (bilateral) & & 0,000 \\
& $\mathrm{~N}$ & 655 & 572 \\
\hline \multirow{3}{*}{ Gastronomía } & Correlación de Pearson & $0,292^{* *}$ & 1 \\
& Sig. (bilateral) & 0,000 & 590 \\
& $\mathrm{~N}$ & 572 & \\
\hline
\end{tabular}

**. La correlación es significativa al nivel 0,01 (bilateral).

Fuente: Elaboración propia. 


\section{IV.2. Productos típicos de la gastronomía extremeña y hábitos de consumo}

La identificación de Extremadura como un destino de turismo gastronómico depende, de manera ineludible, de la calidad y de la peculiaridad de sus platos y alimentos típicos, capaces de diferenciar su gastronomía local. Aun así, la promoción y la difusión de los mismos se hace imprescindible para garantizar su éxito. Por esta razón, se han medido los niveles de conocimiento de los productos y platos de la cocina local, así como la cuantificación de su valoración. Dentro de las reflexiones relativas a los niveles de publicidad y popularidad que caracterizan la gastronomía extremeña, debe incluirse también una consideración acerca de los hábitos de consumo de productos más representativos y elaborados en la región. Los resultados relativos a estos aspectos se detallan en la Tabla 8.

Tabla 8

PREFERENCIAACERCA DE LOS PRODUCTOS TÍPICOS Y HÁBITOS DE CONSUMO

\begin{tabular}{|c|c|c|c|c|}
\hline Variable & \multicolumn{2}{|c|}{ Ítem } & Frecuencia & $\%$ \\
\hline & \multicolumn{2}{|c|}{ Jamón } & 592 & 82,2 \\
\hline & \multicolumn{2}{|c|}{ Torta del Casar } & 544 & 75,6 \\
\hline & \multicolumn{2}{|c|}{ Frite extremeño } & 142 & 19,7 \\
\hline Platos o productos que conoce & \multicolumn{2}{|c|}{ Prueba de cerdo } & 272 & 37,8 \\
\hline & \multicolumn{2}{|c|}{ Carne de caza } & 209 & 29 \\
\hline & \multicolumn{2}{|c|}{ Migas } & 459 & 63,8 \\
\hline & \multicolumn{2}{|c|}{ Otros } & 85 & 11,8 \\
\hline & \multicolumn{2}{|c|}{ Jamón } & 548 & 76,1 \\
\hline & \multicolumn{2}{|c|}{ Torta del Casar } & 363 & 50,4 \\
\hline & \multicolumn{2}{|c|}{ Frite extremeño } & 61 & 8,5 \\
\hline Platos o productos que prefiere & \multicolumn{2}{|c|}{ Prueba de cerdo } & 95 & 13,2 \\
\hline & \multicolumn{2}{|c|}{ Carne de caza } & 68 & 9,4 \\
\hline & \multicolumn{2}{|c|}{ Migas } & 240 & 33,3 \\
\hline & \multicolumn{2}{|c|}{ Otros } & 39 & 5,4 \\
\hline Conocimiento Vinos D.O. Ribera & \multicolumn{2}{|l|}{$\mathrm{Si}$} & 303 & 45,2 \\
\hline del Guadiana & \multicolumn{2}{|l|}{ No } & 367 & 54,8 \\
\hline \multirow{4}{*}{$\begin{array}{l}\text { Consumo y frecuencia de } \\
\text { consumo de productos extremeños } \\
\text { en lugar de residencia }\end{array}$} & $\mathrm{Si}$ & Habitualmente & 200 & 29,3 \\
\hline & & $\begin{array}{l}\text { En esporádicas } \\
\text { ocasiones }\end{array}$ & 290 & 42,5 \\
\hline & & Casi nunca & 87 & 12,8 \\
\hline & \multicolumn{2}{|l|}{$\mathrm{No}$} & 105 & 15,4 \\
\hline
\end{tabular}

Fuente: Elaboración propia.

El jamón y la torta del Casar son los productos más populares ya que el 82,2\% y el 75,6\%, respectivamente, declaran conocerlos. En cambio, el Frite es el plato menos conocido (19,7\%).

En cuanto a las preferencias, los datos corroboran que a mayores niveles de conocimiento de los productos gastronómicos locales corresponden mayores niveles de preferencia. Siguen destacando, pues, el jamón $(76,1 \%)$ y la torta del Casar $(50,4 \%)$. Este resultado nos hace 
entender que existe una importante relación entre la promoción de un producto y el aprecio que le reconoce el consumidor. A la hora de desarrollar una propuesta turística asentada en el patrimonio culinario, es importante tener en cuenta estos datos para poder incidir en ellos en las campañas promocionales. Resulta evidente que es imprescindible emprender acciones de difusión de los demás productos que cualifican la oferta gastronómica local, para que, gracias a su mayor conocimiento, puedan ser apreciados por los turistas al igual que los dos productos estrella: el jamón y la torta del Casar. Así, un mayor conocimiento y consumo de los productos extremeños de calidad debe ir de la mano de una oferta de restauración capaz de poner en valor estos recursos. A este respecto y, especialmente en relación con las carnes selectas de Extremadura, hace falta llevar a cabo una correcta gestión empresarial de la restauración local, con la finalidad de dotarla de restaurantes y asadores capaces de elaborar los productos de la tierra y conservar su autenticidad y carácter autóctono.

Por otro lado, la consideración del enoturismo como uno de los subsectores específicos del turismo gastronómico (Andreu y Verdú, 2012; López-Guzmán et al., 2013; LópezGuzmán y Sánchez-Cañizares, 2008; Rodríguez García et al, 2010) ha llevado a la inclusión en el cuestionario de una pregunta específica relacionada con los niveles de conocimiento que mantiene el turista acerca del vino que se produce en Extremadura y que está amparado por la Denominación de Origen Ribera del Guadiana. Al igual que para los platos y los alimentos típicos, consideramos que unos elevados niveles de popularidad y de promoción son fundamentales también para la difusión de los vinos de la tierra. Estos últimos representan un importante elemento para el turismo gastronómico y son capaces de atraer a un público selecto, cuyo nivel de gasto se sitúa por encima de la media nacional del gasto diario por turista (Mondéjar et al., 2009).

El vino de la Denominación de Origen Ribera del Guadiana no es muy popular entre la demanda turística que visita Extremadura. El porcentaje de turistas que declaran no conocer el vino de esta Denominación es mayor $(54,8 \%)$ que aquéllos que declaran conocerlo $(45,2 \%)$.

Este dato representa seguramente una debilidad para el desarrollo del turismo enogastronómico en Extremadura ya que todo destino turístico que quiera destacar por su patrimonio culinario no puede prescindir de una propuesta enológica de calidad y de reconocido prestigio.

Con la finalidad de averiguar la penetración en los mercados nacionales de los alimentos extremeños y el apego que los turistas mantienen con ellos, eligiéndolos en su cotidianidad, fuera de los límites temporales y geográficos de las vacaciones, se ha medido la costumbre a consumir productos extremeños en los lugares de residencia de los encuestados. Tan sólo el $15,4 \%$ de los encuestados declara no consumir productos extremeños en su vida cotidiana. En cambio, los porcentajes más elevados se registran por aquellos turistas que admiten hacer uso de productos locales habitualmente $(29,3 \%)$ o en esporádicas ocasiones $(42,5 \%)$. Estos resultados ponen de relieve la paulatina notoriedad que están adquiriendo los productos gastronómicos típicos locales.

Analizando las relaciones entre la motivación gastronómica del viaje y los hábitos de consumo de los productos locales, podemos observar que entre los turistas que han reconocido en la gastronomía un elemento muy importante en la elección del destino a visitar, la mayoría $(44,3 \%)$ afirma consumir alimentos extremeños en esporádicas ocasiones y el $30,2 \%$ declara hacer uso de los mismos habitualmente (Véase Tabla 9). Este dato demuestra 
que el aprecio de la gastronomía típica a lo largo de los viajes puede crear un vínculo con el destino que perdura una vez acabada las vacaciones y que sigue repercutiendo positivamente en la economía de la región gracias a los gastos en alimentos extremeños que los turistas realizan en sus ciudades de origen.

Tabla 9

RELACIÓN ENTRE LA FRECUENCIA DE CONSUMO DE PRODUCTOS EXTREMEÑOS Y LAMOTIVACIÓN GASTRONÓMICA DEL VIAJE

\begin{tabular}{|c|c|c|c|c|c|c|c|}
\hline & \multicolumn{4}{|c|}{ Frecuencia en el consumo de productos extremeños } & \multirow[b]{2}{*}{ Total } \\
\hline & & & Habitualmente & $\begin{array}{c}\text { En } \\
\text { esporádicas } \\
\text { ocasiones }\end{array}$ & Casi nunca & No & \\
\hline \multirow{5}{*}{ 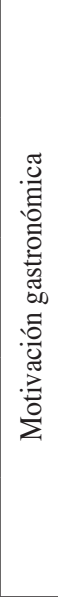 } & $\begin{array}{l}\text { Nada } \\
\text { importante }\end{array}$ & $\begin{array}{l}\text { Recuento } \\
\% \text { dentro de } \\
\text { gastronomía }\end{array}$ & $\begin{array}{r}11 \\
31,4 \%\end{array}$ & $\begin{array}{r}9 \\
25,7 \%\end{array}$ & $\begin{array}{r}5 \\
14,3 \%\end{array}$ & $\begin{array}{r}10 \\
28,6 \%\end{array}$ & $\begin{array}{r}35 \\
100,0 \%\end{array}$ \\
\hline & $\begin{array}{l}\text { Poco } \\
\text { importante }\end{array}$ & $\begin{array}{l}\text { Recuento } \\
\% \text { dentro de } \\
\text { gastronomía }\end{array}$ & $\begin{array}{r}6 \\
25,0 \%\end{array}$ & $\begin{array}{r}6 \\
25,0 \%\end{array}$ & $\begin{array}{r}5 \\
20,8 \%\end{array}$ & $\begin{array}{r}7 \\
29,2 \%\end{array}$ & $\begin{array}{r}24 \\
100,0 \%\end{array}$ \\
\hline & Importante & $\begin{array}{l}\text { Recuento } \\
\% \text { dentro de } \\
\text { gastronomía }\end{array}$ & $\begin{array}{r}32 \\
27,8 \%\end{array}$ & $\begin{array}{r}47 \\
40,9 \%\end{array}$ & $\begin{array}{r}13 \\
11,3 \%\end{array}$ & $\begin{array}{r}23 \\
20,0 \%\end{array}$ & $\begin{array}{r}115 \\
100,0 \%\end{array}$ \\
\hline & $\begin{array}{l}\text { Bastante } \\
\text { importante }\end{array}$ & $\begin{array}{l}\text { Recuento } \\
\% \text { dentro de } \\
\text { gastronomía }\end{array}$ & $\begin{array}{r}49 \\
26,5 \%\end{array}$ & $\begin{array}{r}93 \\
50,3 \%\end{array}$ & $\begin{array}{r}19 \\
10,3 \%\end{array}$ & $\begin{array}{r}24 \\
13,0 \%\end{array}$ & $\begin{array}{r}185 \\
100,0 \%\end{array}$ \\
\hline & $\begin{array}{l}\text { Muy } \\
\text { importante }\end{array}$ & $\begin{array}{l}\text { Recuento } \\
\% \text { dentro de } \\
\text { gastronomía }\end{array}$ & $\begin{array}{r}65 \\
30,2 \%\end{array}$ & $\begin{array}{r}95 \\
44,2 \%\end{array}$ & $\begin{array}{r}29 \\
13,5 \%\end{array}$ & $\begin{array}{r}26 \\
12,1 \%\end{array}$ & $\begin{array}{r}215 \\
100,0 \%\end{array}$ \\
\hline \multicolumn{2}{|c|}{ Total } & $\begin{array}{l}\text { Recuento } \\
\% \text { dentro de } \\
\text { gastronomía }\end{array}$ & $\begin{array}{r}163 \\
28,4 \%\end{array}$ & $\begin{array}{r}250 \\
43,6 \%\end{array}$ & $\begin{array}{r}71 \\
12,4 \%\end{array}$ & $\begin{array}{r}90 \\
15,7 \%\end{array}$ & $\begin{array}{r}574 \\
100,0 \%\end{array}$ \\
\hline
\end{tabular}

Fuente: Elaboración propia.

Finalmente cabe destacar que los alimentos típicos de Extremadura reciben una elevada evaluación por parte de los turistas en relación con los niveles de calidad. Los resultados pueden apreciarse en la Tabla 10, donde 1 corresponde a una calidad muy baja y 5 a excelente.

Es interesante ver que la casi totalidad de los productos reciben una evaluación superior al 4 en una escala Likert de 5 puntos. Este dato nos comunica que los alimentos típicos extremeños están bien posicionados en la mente de los consumidores turísticos. Su buena reputación representa un importante punto de partida para la elaboración de una propuesta turística centrada en los patrimonios gastronómicos locales. Llama la atención la evaluación del vino de la Denominación de Origen Ribera del Guadiana que se coloca como la más baja $(3,79)$, con respecto a las de los demás alimentos. Este resultado podría ser debido tanto a una efectiva carencia en los niveles de calidad de los vinos de la tierra como, sobre todo, a una escasa promoción y divulgación de los mismos. En ambos casos, los resultados de la investigación demandan la necesidad de acciones correctivas al respecto. 


\section{IV.3. Satisfacción con la gastronomía local y con la visita}

El conocimiento de los elementos de un destino o de un determinado producto turístico que determina mayores o menores niveles de satisfacción del viajero es algo crucial para el marketing y la planificación estratégica y, en consecuencia, para la eficiencia y el éxito de un destino turístico (Severtet al., 2007; Yoon y Uysal, 2005).

La satisfacción del consumidor representa el principal feedback de todo tipo de industria. En esta investigación la evaluación de todos los aspectos relacionados con la experiencia gastronómica vivida a lo largo de la estancia en Extremadura ha ocupado un espacio importante. Tres de las 18 preguntas del cuestionario han sido encaminadas a medir la satisfacción general que tiene el turista con respecto a su experiencia turística, a la gastronomía local y a algunos aspectos específicos de la misma. Se ha adoptado una escala de medición Likert de 5 puntos. Los principales resultados se pueden observar en la Tabla 11.

Tabla 11

SATISFACCIÓN CON LA GASTRONOMÍA Y LA VISITA DE LA CIUDAD DE CÁCERES

\begin{tabular}{|c|l|c|c|c|c|}
\hline Variable & \multicolumn{1}{|c|}{ Ítem } & Media & Min & Max & Desv. Tip. \\
\hline \multirow{4}{*}{$\begin{array}{l}\text { Satisfacción } \\
\text { especifica }\end{array}$} & Gastronomía tradicional & 4,21 & 1 & 5 & 0,897 \\
\cline { 2 - 6 } & Servicio y hospitalidad & 4,13 & 1 & 5 & 0,868 \\
\cline { 2 - 6 } & Calidad platos & 3,97 & 1 & 5 & 0,859 \\
\cline { 2 - 6 } & Ambiente establecim. & 3,83 & 1 & 5 & 0,927 \\
\cline { 2 - 6 } & Instalaciones & 3,80 & 1 & 5 & 0,899 \\
\cline { 2 - 6 } & Innovación y nuevos sabores & 3,55 & 1 & 5 & 1,044 \\
\cline { 2 - 6 } & Precios & 3,51 & 1 & 5 & 0,922 \\
\hline \multirow{2}{*}{$\begin{array}{l}\text { Satisfacción } \\
\text { general }\end{array}$} & Visita & 4,34 & 1 & 5 & 0,709 \\
\cline { 2 - 6 } & Gastronomía & 4,10 & 1 & 5 & 0,815 \\
\hline
\end{tabular}

Fuente: Elaboración propia.

Podemos ver que el estudio evidencia niveles elevados de aprecio y satisfacción del turista en relación con la gastronomía local. Resulta interesante detenernos en los niveles de satisfacción con la gastronomía local alcanzados por los denominados turistas foodies (Fox, 2007), que en esta investigación coinciden con aquellos viajeros para los cuáles la motivación gastronómica ha sido importante para la elección del destino.

En la Tabla 12 se puede apreciar la relación que existe entre la motivación gastronómica y los niveles de satisfacción alcanzados por el turista en relación con la experiencia culinaria vivida.

Finalmente, podemos concluir que, en media, la satisfacción de los turistas con la gastronomía local es de 4,1 en una escala Likert de 5 puntos. A nivel cuantitativo, destacar que el $46,1 \%$ de los encuestados declara estar bastante satisfecho con la gastronomía local y el $33,5 \%$ muy satisfecho. Asimismo, debemos reconocer que, en relación con la muestra considerada, la repetición de la visita no influye de manera tajante en los niveles de satisfacción, que son elevados tanto en los turistas que visitan Cáceres por primera vez como en aquéllos que ya tenían una experiencia previa de la ciudad. 
Tabla 12

RELACIÓN ENTRE LAMOTIVACIÓN GASTRONÓMICA DEL VIAJE Y

LA SATISFACCIÓN CON LA GASTRONOMÍA DEL DESTINO

\begin{tabular}{|c|c|c|c|c|c|c|c|c|}
\hline & \multicolumn{5}{|c|}{ Satisfacción con la gastronomía } & \multirow[t]{2}{*}{ Total } \\
\hline & & & $\begin{array}{c}\text { Muy } \\
\text { insatisfecho }\end{array}$ & $\begin{array}{c}\text { Poco } \\
\text { satisfecho }\end{array}$ & Satisfecho & $\begin{array}{c}\text { Bastante } \\
\text { satisfecho }\end{array}$ & $\begin{array}{c}\text { Muy } \\
\text { satisfecho }\end{array}$ & \\
\hline \multirow{5}{*}{ 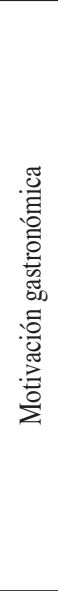 } & $\begin{array}{l}\text { Nada } \\
\text { importante }\end{array}$ & $\begin{array}{l}\text { Recuento } \\
\text { \% dentro de } \\
\text { gastronomía } \\
\end{array}$ & $\begin{array}{r}0 \\
0,0 \%\end{array}$ & $\begin{array}{r}2 \\
6,1 \%\end{array}$ & $\begin{array}{r}9 \\
27,3 \%\end{array}$ & $\begin{array}{r}8 \\
24,2 \%\end{array}$ & $\begin{array}{r}14 \\
42,4 \%\end{array}$ & $\begin{array}{r}33 \\
100,0 \%\end{array}$ \\
\hline & $\begin{array}{l}\text { Poco } \\
\text { importante }\end{array}$ & $\begin{array}{l}\text { Recuento } \\
\text { \% dentro de } \\
\text { gastronomía } \\
\end{array}$ & $\begin{array}{r}0 \\
0,0 \%\end{array}$ & $\begin{array}{r}1 \\
4,3 \% \\
\end{array}$ & $\begin{array}{r}11 \\
47,8 \%\end{array}$ & $\begin{array}{r}8 \\
34,8 \%\end{array}$ & $\begin{array}{r}3 \\
13,0 \% \\
\end{array}$ & $\begin{array}{r}23 \\
100,0 \%\end{array}$ \\
\hline & Importante & $\begin{array}{l}\text { Recuento } \\
\text { \% dentro de } \\
\text { gastronomía }\end{array}$ & $\begin{array}{r}1 \\
0,9 \%\end{array}$ & $\begin{array}{r}3 \\
2,8 \%\end{array}$ & $\begin{array}{r}34 \\
31,8 \%\end{array}$ & $\begin{array}{r}49 \\
45,8 \%\end{array}$ & $\begin{array}{r}20 \\
18,7 \%\end{array}$ & $\begin{array}{r}107 \\
100,0 \%\end{array}$ \\
\hline & $\begin{array}{l}\text { Bastante } \\
\text { importante }\end{array}$ & $\begin{array}{l}\text { Recuento } \\
\text { \% dentro de } \\
\text { gastronomía }\end{array}$ & $\begin{array}{r}3 \\
1,7 \%\end{array}$ & $\begin{array}{r}1 \\
0,6 \%\end{array}$ & $\begin{array}{r}27 \\
15,3 \%\end{array}$ & $\begin{array}{r}106 \\
59,9 \%\end{array}$ & $\begin{array}{r}40 \\
22,6 \%\end{array}$ & $\begin{array}{r}177 \\
100,0 \%\end{array}$ \\
\hline & $\begin{array}{l}\text { Muy } \\
\text { importante }\end{array}$ & $\begin{array}{l}\text { Recuento } \\
\text { \% dentro de } \\
\text { gastronomía }\end{array}$ & $\begin{array}{r}1 \\
0,5 \%\end{array}$ & $\begin{array}{r}0 \\
0,0 \%\end{array}$ & $\begin{array}{r}14 \\
6,8 \%\end{array}$ & $\begin{array}{r}82 \\
39,6 \%\end{array}$ & $\begin{array}{r}110 \\
53,1 \% \\
\end{array}$ & $\begin{array}{r}207 \\
100,0 \%\end{array}$ \\
\hline \multicolumn{2}{|l|}{ Total } & $\begin{array}{l}\text { Recuento } \\
\text { \% dentro de } \\
\text { gastronomía }\end{array}$ & $\begin{array}{r}5 \\
0,9 \%\end{array}$ & $\begin{array}{r}7 \\
1,3 \%\end{array}$ & $\begin{array}{r}95 \\
17,4 \%\end{array}$ & $\begin{array}{r}253 \\
46,3 \%\end{array}$ & $\begin{array}{r}187 \\
34,2 \% \\
\end{array}$ & $\begin{array}{r}547 \\
100,0 \%\end{array}$ \\
\hline
\end{tabular}

Fuente: Elaboración propia.

\section{CONCLUSIONES}

Las asociaciones entre cultura y gastronomía son fundamentales si pensamos en el potencial de diversificación y especialización que mantienen para la oferta de las ciudades culturales o de interior. Una propuesta culinaria eficaz y bien estructurada podría ser un elemento capaz de aumentar las pernoctaciones de los viajeros y representar un elemento de fidelización del mismo.

El principal objetivo de este artículo ha sido la identificación de una tendencia emergente dentro de la demanda turística que visita Extremadura y que va reconociendo un papel cada vez mayor a los patrimonios gastronómicos locales. En estos últimos identifica un vehículo de la cultura de un destino.

El estudio teórico de la literatura científica analizada ha puesto de manifiesto que el turismo gastronómico debe entenderse dentro de la categoría más amplia y consolidada del turismo cultural. Los productos alimentarios típicos, así como los platos y las recetas tradicionales de un destino, son la expresión de la historia, la religión, el recorrido social y político de un territorio. Conocerlos y degustarlos significa acercarse a un determinando contexto social y cultural, posiblemente muy diferente al de la cotidianidad de cada uno. 
Gracias al estudio empírico realizado, hemos tenido la oportunidad de obtener unos resultados que nos permiten sacar algunas importantes conclusiones acerca del interés que mantienen los turistas que viajan a Extremadura hacía su gastronomía tradicional.

En primer lugar, hemos podido conocer la demanda de turismo gastronómico que recibe la ciudad de Cáceres y trazar su perfil sociodemográfico. Podemos concluir que se trata de un viajero maduro, cuya edad se coloca entre los 40 y los 49 años. La nacionalidad de los viajeros es principalmente española y tan sólo un 9,2\% procede del extranjero.

En cuanto al nivel de formación, la mayor parte de los encuestados $(57,5 \%)$ declara tener estudios universitarios y, por lo tanto, un nivel cultural alto. Se trata de un segmento con un poder adquisitivo elevado, destacando aquellos viajeros con unos ingresos mensuales superiores a los 2.000 euros. La estancia media es breve. La mayor parte de los encuestados ha declarado pasar sólo una noche en el destino. Este dato pone de relieve la necesidad de retener más a los turistas, para que su estancia sea más larga. Una propuesta gastronómica compleja e integrada, que no se limite sólo a la degustación de un menú típico, sino que abarque una experiencia sensorial y cultural amplia, puede representar una posibilidad creíble.

La satisfacción media de los turistas en relación con la gastronomía local se registra en 4,10 puntos en una escala Likert de 5 puntos, donde 1 corresponde a muy insatisfecho y 5 a muy satisfecho. Los resultados subrayan la necesidad de mejorar algunos aspectos de la oferta gastronómica local como la innovación y la propuesta de nuevos platos y sabores (valoración media=3,55). En general, la satisfacción global con la visita se ha registrado en 4,34 puntos.

En este artículo se ha reconocido una particular importancia del papel que juega la gastronomía como factor motivacional determinante para la elección del viaje. Los resultados apuntan que más del $50 \%$ de los encuestados ha declarado que la gastronomía ha sido un elemento muy importante o bastante importante en la elección de Cáceres como destino de vacaciones. No obstante, la motivación más destacada coincide con la cultural. La curiosidad de conocer la cultura y los patrimonios de la ciudad no debe sorprender considerando que la principal vocación turística de este destino es cultural. Es interesante, en cambio, observar las relaciones que subyacen entre estos dos factores motivacionales. Pese a ser la gastronomía la segunda motivación más importante, siendo la cultural la primera, podemos ver que existe una correlación positiva entre las dos variables analizadas $(r=0,292 ; p=0,000)$. La principal conclusión de este estudio debe identificarse en la demostrada correlación que une los dos factores motivacionales considerados. Normalmente, si para un turista es importante la cultura de un destino lo es también la gastronomía. Este resultado es una importante ayuda para el futuro enfoque de la propuesta cultural de los destinos de interior y urbanos, que tradicionalmente asientan su actividad turística en los patrimonios culturales locales. La valorización de los recursos culinarios y gastronómicos a través de productos claros y estructurados podría aportar un valor añadido a los destinos que aumentarían su competitividad y sostenibilidad en los mercados nacionales e internacionales.

\section{BIBLIOGRAFÍA}

ACEVIN (2012): Informe de visitantes a bodegas asociadas a las Rutas del Vino de España. Año 2011. Ministerio de Industria Energía y Turismo e Instituto de Turismo de España. Madrid. 
ANDREU GUERRERO, R. y VERDÚ ALBERT, L. (2012). «Turismo enológico en Alicante: la ruta del vino en el municipio de Pinoso». Cuadernos de Turismo, $\mathrm{n}^{\circ}$ 30, 35-61.

ÁVILA, R. y BARRADO, D.A. (2005): «Nuevas tendencia en el desarrollo de destinos turísticos: marcos conceptuales y operativos para su planificación y gestión». Cuadernos de Turismo, $\mathrm{n}^{\circ} 15,27-43$.

BELISLE, F.J. (1983): «Tourism and food production in the Caribbean». Annals of Tourism Research, $\mathrm{n}^{\circ}$ 4, 497-513.

CALZATI, V. (2009): «I territori lenti: definizione e caratteri» en Sviluppo turistico e territori lenti (CST, Coord.). Milano, Edit. Franco Angeli, 15-30.

CAMPÓN, A.M., FOLGADO, J.A. y HERNÁNDEZ, J.M. (2011): «Turismo gastronómico: una revisión de la literatura en España», Comunicación presentada en el XIII Seminario Luso-Espanhol de Economía Empresarial, celebrado en la Universidad de Évora, 24-25 de noviembre.

DÍAZ ARMAS, R.J. (2008): «Potencialidad e integración del turismo del vino en un destino de sol y playa: el caso de Tenerife». Revista Pasos, vol. 6 (2), pp. 199-212.

DOS SANTOS, R. J. y ANTONINI, B.O. (2004): «La gastronomía típica de la Isla de Santa Catarina (Brasil)». Estudios y Perspectivas en Turismo, $\mathrm{n}^{\circ}$ 13, 89-110.

ESPEITX, E. (2004): «Patrimonio alimentario y turismo: una relación singular». Pasos, Revista de Turismo y Patrimonio Cultural, $\mathrm{n}^{\circ} 2$, 193-213.

FAULKNER, B., OPPERMAN, M. y FREDLINE, E. (1999): «Destination competitiveness: an exploratory examination of South Australia's core attractions». Journal of Vacation Marketing, $\mathrm{n}^{\circ}$ 2, 125-139.

FEO, F. (2005): «Turismo gastronómico en Asturias». Cuadernos de Turismo, $\mathrm{n}^{\circ}$ 15, 77-96.

FOLGADO, J. A., HERNÁNDEZ, J. M y CAMPÓN, A. M. (2011): «El turismo gastronómico como atractivo innovador del destino: un estudio empírico sobre rutas gastronómicas españolas».Comunicación presentada en el XIII Seminario Luso-Espanhol de Economía Empresarial, celebrado en la Universidad de Évora, 24-25 de noviembre.

FOX, R. (2007): «Reinventing the gastronomic identity of Croatian tourist destinations». International Journal of Hospitality Management, $\mathrm{n}^{\circ} 3,546-559$.

GUÍA MICHELIN (2012): España \& Portugal 2012. Madrid. Michelin.

GUÍA REPSOL (2012): Guía Repsol, Turismo con los mejores Restaurantes. Madrid. Repsol YPF S.A.

HALL, C. M. y SHARPLES, L. (2003): «The consumption of experiences or the experiences of consumption? An introduction to the tourism of taste» en Food tourism around the world: Development, management and markets (Hall, C. M., Sharples, E., Mitchell, R., Macionis, N. y Cambourne, B., Coord.). Oxford, Edit. Butterworth-Heinemann, 1-24.

HJALAGER, A.M. (2010): «A review of innovation research in tourism». Tourism Management, $\mathrm{n}^{\circ} 1,1-12$.

HJALAGER, A. y RICHARDS, G. (2002): Tourism and gastronomy. London. Routledge.

INE (2012): Encuesta de ocupación hotelera (2007-2011). Madrid. Instituto Nacional de Estadística.

KIVELA, J. y CROTTS, J. (2006): «Tourism and gastronomy: gastronomy's influence on how tourists experience a destination». Journal of Hospitality \& Tourism Research, $\mathrm{n}^{\mathrm{o}} 3$, 354-377. 
LANCERINI, E. (2005): «Territori Lenti: Contributi per una nuova geografia dei paesaggi abitati italiani». Territorio, $\mathrm{n}^{\circ}$ 34, 9-15.

LANZANI, A. (2005): «Geografie, paesaggi, pratiche dell'abitare e progetti di sviluppo». Territorio, $\mathrm{n}^{\circ} 34,19-37$.

LEAL, M.P. (2011): «La diversificación del destino turístico a través del turismo gastronómico: el caso de Vilanova i la Geltrú (Barcelona)». Pasos, Revista de Turismo y Patrimonio Cultural, $\mathrm{n}^{\mathrm{o}} 1,15-24$.

LEE, T. y CROMPTON, J. (1992): «Measuring novelty seeking in tourism». Annals of Tourism Research, $\mathrm{n}^{\circ}$ 4, 732-737.

LONG, L. (2003): Culinary tourism. Lexington. University of Kentucky Press.

LÓPEZ-GUZMÁN, T.J., RODRÍGUEZ GARCÍA, J. y VIEIRA RODRÍGUEZ, A. (2013) «Revisión de la literatura científica sobre enoturismo en España».Cuadernos de turismo, no32, 171-188.

LÓPEZ-GUZMÁN, T.J. y SÁNCHEZ-CAÑIZARES, S.M. (2008): «La creación de productos turísticos utilizando rutas enológicas». Pasos, Revista de Turismo y Patrimonio Cultural, $\mathrm{n}^{\circ} 2,159-171$.

MAK, A. H. N., LUMBERS, M. y EVES, A. (2012a): «Factors influencing tourist food consumption». International Journal of Hospitality Management, $\mathrm{n}^{\circ}$ 3, 928-936.

MAK, A. H. N., LUMBERS, M. y EVES, A. (2012b): «Globalisation and food consumption in tourism».Annals of TourismResearch, $\mathrm{n}^{\circ}$ 1, 171-196.

MILLÁN, M.G. y AGUDO, E.M. (2010): «El turismo gastronómico y las Denominaciones de Origen en el sur de España: Oleoturismo. Un estudio de caso». Pasos, Revista de Turismo y Patrimonio Cultural, $\mathrm{n}^{\circ} 1,91-112$.

MILLÁN, M.G., PÉREZ, L.M. y MORALES, E. (2010): «La Ruta del Aceite de Oliva en la DOP Baena como opción estratégica de desarrollo». Revista de Economía, Sociedad Turismo y Medioambiente, RESTMA, $\mathrm{n}^{\circ}$ 10, 33-52.

MOLZ, J.G. (2007): «Eating Difference: The Cosmopolitan Mobilities of CulinaryTourism». Space and Culture, $\mathrm{n}^{\circ}$ 1, 77-93.

MONDÉJAR, J.A., VARGAS, M., CORDENTE, M. y CARRICANO, M. (2009): «Nuevos productos turísticos: estrategias de enoturismo en Castilla-La Mancha» en Principales tendencias de investigación en turismo (Ferrari, G., Mondéjar, J., Mondéjar, J.A., Vargas, M., Coord.). Oviedo, Edit. Septem Ediciones, 117-134.

ORTEGA, F.J., SÁNCHEZ, J.M. y HERNÁNDEZ, J.M. (2012): La gastronomía «de alta gama, de lujo o de calidad»como eje potenciador del turismo cultural y de sensaciones en Extremadura. Cáceres. Fundación Caja Extremadura.

QUAN, S. y WANG, N. (2004): «Towards a structural model of tourist experience: an illustration from food experiences in tourism». Tourism Management, $\mathrm{n}^{\circ} 3$, 297-305.

RAMOS, V., RAY-MAQUIEIRA, J. y TUGORES, M. (2004): The role of training in changing an economy specialising in tourism. International Journal of Manpower, $\mathrm{n}^{\circ} 1,55-72$.

REYNOLDS, P.C. (1993): «Food and tourism: towards an understanding of sustainable culture». Journal of Sustainable Tourism, $\mathrm{n}^{\circ}$ 1, 48-54.

RICHARDS, G. (2002): «Gastronomy: An essential ingredient in tourism production and consumption? » en Tourism and gastronomy (Hjalager, A.-M. y Richards, G., Coord.). London, Edit. Routledge, 3-20. 
RITZER, G. (1995): The McDonaldization of society. London. Sage.

RODEN, C. (2003): «Local food and culture» en Local Food \& Tourism International Conference: Larnaka, Cyprus 9-11 November 2000 (OMT, Coord.). Madrid, Edit. World Tourism Organization, 9-14.

RODRÍGUEZ GARCÍA, J., LÓPEZ-GUZMÁN, T.J., CAÑIZARES RUÍZ, S.M. y JIMÉNEZ GARCÍA, M. (2010). «Turismo del vino en el marco de Jerez. Un análisis desde la perspectiva de la oferta». Cuadernos de Turismo, n 26, 217-234.

SÁNCHEZ-CAÑIZARES, S. y LÓPEZ-GUZMÁN, T. (2011): «Gastronomy as a tourism resource: profile of the culinary tourist». CurrentIssues in Tourism, $\mathrm{n}^{\circ} 3,229-245$.

SCHLÜTER, R.G. (2009): «Turismo gastronómico y medioambiente: en busca de la sustentabilidad» en Principales tendencias de investigación en turismo (Ferrari, G., Mondéjar, J., Mondéjar, J.A., Vargas, M., coord.). Oviedo, Edit. Septem Ediciones, 43-62.

SEGITTUR (2007): Análisis de la demanda de Turismo enogastronómico en España.Madrid. Ministerio de Industria, Turismo y Comercio.

SEVERT, D., WANG, Y., CHEN, P. y BREITER, D. (2007): «Examining the motivation, perceived performance, and behavioural intentions of convention attendees: Evidence from a regional conference». Tourism Management, $\mathrm{n}^{\circ}$ 2, 399-408.

SIDALI, K.L., KASTENHOLZ, E., BIANCHI, R. (2013): «Food tourism, niche markets and products in rural tourism: combining the intimacy model and the experience economy as a rural development strategy». Journal of Sustainable Tourism, DOI: dx.doi.org/10.1080 /09669582.2013.836210

STEWART, J., BRAMBLE, L. y ZIRALDO, D. (2008): «Key challenges in wine and culinary tourism with practical recommendations». International Journal of Contemporary Hospitality Management, $\mathrm{n}^{\mathrm{o}}$ 3, 302-312.

TELFER, D. J. y WALL, G. (2000): «Strengthening backward economic linkages: Local food purchasing by three indonesian hotels». Tourism Geographies, no 4, 421-447.

YOON, Y. y UYSAL, M. (2005): «An examination of the effects of motivation and satisfaction on destination loyalty: a structural model», Tourism Management, $\mathrm{n}^{\mathrm{o}}$ 1, 45-56. 
